\title{
Fetal ventriculomegaly: Pregnancy outcomes and follow-ups in ten years
}

\author{
Nan Chu ${ }^{1,2}$, Yueping Zhang ${ }^{1}$, Yingliu Yan ${ }^{1}$, Yunyun Ren ${ }^{1}$, Ling Wang ${ }^{1,2,3,4,}$, Bin Zhang ${ }^{1,2, *}$ \\ ${ }^{1}$ Obstetrics and Gynecology Hospital of Fudan University, Shanghai, China; \\ ${ }^{2}$ Shanghai Key Laboratory of Female Reproductive Endocrine Related Diseases, Shanghai, China; \\ ${ }^{3}$ Laboratory for Reproductive Immunology, Hospital \& Institute of Obstetrics and Gynecology, IBS, Fudan University Shanghai \\ Medical College, Shanghai, China; \\ ${ }^{4}$ The Academy of Integrative Medicine of Fudan University, Shanghai, China.
}

\begin{abstract}
Summary The aim of this study is to evaluate the pregnancy outcomes and prognoses for fetuses with ventriculomegaly. Two hundred and forty-one cases of fetuses with ventriculomegaly were included in this study. The subjects were divided into three groups according to their lateral ventricular width: "Mild Ventriculomegaly" (10 - < $12 \mathrm{~mm})$, "Moderate Ventriculomegaly" $(12-<15 \mathrm{~mm})$ and "Severe Ventriculomegaly" $(\geq 15 \mathrm{~mm})$. Pediatric examination records and telephone interviews were conducted to track the outcomes of children until the age of 9 years. Eight-two cases were Isolated Ventriculomegaly (34.0\%), while Non-Isolated Ventriculomegaly was found in 159 cases $(66.0 \%)$. The pregnancy was terminated in 91 cases, and a higher abortion ratio was found in the NIVM (Non-Isolated Ventriculomegaly) group compared with the IVM (Isolated Ventriculomegaly) group. The fetuses were delivered in 150 cases, and four infants suffered deaths with NIVM. Of the surviving fetuses, 7 with IVM and 9 with NIVM showed significant abnormalities. The Mild and Moderate VM groups had more favorable prognoses compared with the Severe VM group. Regarding the outcomes and progression of lateral ventricular width, 1 out of 42 cases in the regressed group and 19 out of 108 cases in the stable group showed significant abnormalities. This study suggests that the degree and the progression of ventricular dilatation are main factors that affect pregnancy outcomes and prognoses.
\end{abstract}

Keywords: Prenatal diagnosis, ventriculomegaly, pregnancy outcome

\section{Introduction}

Ventriculomegaly is one of the most common cerebral findings on obstetrical ultrasound scans during prenatal examinations (1). Ventriculomegaly is diagnosed when atrial diameter of one or both lateral ventricles is greater than $10 \mathrm{~mm}$ on an ultrasound examination performed between 15 and 40 weeks of gestation. Based on the width of the fetus's lateral ventricles observed during the ultrasound examination, some studies (2) usually

Released online in J-STAGE as advance publication April 15, 2016.

*Address correspondence to:

Dr. Ling Wang and Dr. Bin Zhang, Obstetrics \& Gynecology Hospital of Fudan University, 413 Zhaozhou Road, Shanghai 200011, China.

E-mail: Dr.wangling@fudan.edu.cn (LW), shentuzhangbin@163. com (BZ) consider "mild ventriculomegaly" (10-15 mm) and "severe ventriculomegaly" ( $\geq 15 \mathrm{~mm}$ ). Although others have used the categories of mild (10-12 mm), moderate (13-15 mm), and severe ( $\geq 15 \mathrm{~mm})$ (3), considering the different prognoses associated with those widths.

The incidence of ventriculomegaly reported in the literature is between $0.3-2 \%(4,5)$. Fetal cerebral ventriculomegaly is a descriptive diagnosis of symptoms that can result from numerous causes, such as obstructions or congenital infections; and in some cases, no cause can be determined during or even after the pregnancy. Ventriculomegaly can be unilateral or bilateral. It is generally described as "Isolated Ventriculomegaly" if the fetus had no other associated anomalies. The prognoses of fetuses with ventriculomegaly are controversial because of the large variations in study scales and follow-up criteria. Even though some studies had focused on it, the answer is still uncertain. 
The aim of this study was to evaluate pregnancy outcomes and prognoses of fetuses with ventriculomegaly and try to find relevant factors that affect postnatal development.

\section{Materials and Methods}

This research was approved by Ethics Committee at Obstetrics and Gynecology Hospital of Fudan University and written informed consent was obtained from all study participants. All subjects with ventriculomegaly seen at the Multidisciplinary Consultation Center, Obstetrics and Gynecology Hospital, Fudan University between January 2004 and December 2013 were included in this study. According to the width of the fetal lateral ventricles, the subjects were divided into 3 groups: "Mild Ventriculomegaly" (10.0 - < $12.0 \mathrm{~mm})$, "Moderate Ventriculomegaly $(12.0-<15.0 \mathrm{~mm})$, and "Severe Ventriculomegaly" ( $\geq 15.0 \mathrm{~mm}$ ). After ventriculomegaly was diagnosed, another complete sonographic examination was performed to exclude associated intracranial and extracranial anomalies of fetuses. The fetuses were also classified as "Isolated Ventriculomegaly" (IVM) if no associated anomaly was detected at the initial ultrasonography with negative findings in TORCH screening and karyotype examinations. "Non-Isolated Ventriculomegaly" (NIVM) was defined by ultrasonic findings of ventriculomegly with other structural abnormalities or positive findings in TORCH screenings or karyotype examinations.

Additional ultrasound scans were performed every 2-4 weeks until delivery to detect changes in ventricular width and other anomalies. The ultrasound scans were carefully performed by experienced senior ultrasound operators who had the license of prenatal diagnosis with GE Voluson 730 expert transabdominally. Serum screening for Down syndrome, TORCH (toxoplasmosis, other, rubella, cytomegalovirus (CMV) and herpes simplex virus) and fetal karyotype examinations were recommended for all the patients and performed only with the parents' agreement after they were informed of the importance of such testing. The medical records of all cases were carefully reviewed, including the mothers' age, the gestational age and the parents' other characteristics, etc. The pregnancy outcomes were available in all cases.

During the follow-ups of living children, the information about children was collected from their local pediatricians. The children's neurological and developmental status were carefully examined by their pediatricians at six weeks of life and at their following regular visits to pediatric hospitals. The results that evaluate the children's growth, locomotor activities, coordination, hearing and visual function, speech and socialization capacities, neurodevelopmental anomalies, other abnormalities and subsequent treatments were recorded. We also used telephone interviews with the parents as a supplement to the follow-up data. The children's statuses were determined on the basis of pediatric examinations and parental interviews when the child was 1 month, 3 months, 6 months and almost to 9 years old. Significant abnormities were defined as neonatal deaths; structural malformations; poor locomotor, speech or socialization skills; abnormal hearing or visual function; and neurodevelopmental or other anomalies.

SPSS 15.0 (SPSS Inc., Chicago, IL, USA) was used to analyze the data. The chi-square test and Fisher's exact test were used, and significant differences were considered when $p<0.05$. Descriptive statistics were also used to describe the ultrasonography details and follow-up characteristics of all cases.

\section{Results}

From January 2004 to December 2013, 304 women received consultation for fetal ventriculomegaly at the Multidisciplinary Consultation Center. Sixty-three cases were lost to follow-up and the rate of follow up lost was $20.7 \%$ (63/304). Three cases refused to participate in the interviews because of the disruptions that they caused, and 60 cases failed to provide current contact information. The details of patients were summarized as supporting information (Supplemental Data Table S1- S7, http://www.biosciencetrends.com/docindex. php? year $=2016$ \& kanno $=2$ ).

Of the 241 cases that began the study, 151 were diagnosed with "Mild Ventriculomegaly" (62.7\%), 56 were diagnosed with "Moderate Ventriculomegaly" $(23.2 \%)$ and 34 were diagnosed with "Severe Ventriculomegaly" (14.1\%). The mean maternal age in this study was 28 years old (range from 19-44), and the children's gestational age at first diagnosis was 27 weeks (ranged from 18-36 weeks) and most of the ventriculomegaly cases were diagnosed between 24 and 32 weeks. The majority of the mothers were uniparous; the multiple pregnancy rate was $1.6 \%(4 / 241)$, but only one of the twins was affected. In 16 cases, the mothers had an adverse pregnancy history; some of the women experienced spontaneous abortion, and 10 mothers had a history of infantile hydrocephalus. The characteristics of the mothers in the study are summarized in Table 1.

The mean atrium widths of the left ventricles in the Mild Group, Moderate Group and Severe Group were $9.87 \mathrm{~mm}$ (range: $3.6-11.9 \mathrm{~mm}), 12.39 \mathrm{~mm}$ (range: 8.3-14.9 mm), $16.56 \mathrm{~mm}(8-42.8 \mathrm{~mm})$ respectively, and $11.41 \mathrm{~mm}$ (range: $3.6-42.8 \mathrm{~mm}$ ) in total. The mean atrium widths of the right ventricles in the Mild Group, Moderate Group and Severe Group were 9.04 mm (range: 4.1-11.9 mm), $11.21 \mathrm{~mm}$ (range: 3.4$14.4 \mathrm{~mm}$ ), $17.17 \mathrm{~mm}$ (range: 7-37.9 mm) respectively, and $10.69 \mathrm{~mm}$ (range: $3.4-37.9 \mathrm{~mm}$ ) in total. Of the 241 ventriculomegaly cases, 143 were unilateral $(59.3 \%)$, while 98 were bilateral $(40.7 \%)$. The clinical 
Table 1. Characteristics of the mothers and fetuses in the study

\begin{tabular}{|c|c|c|c|c|c|c|}
\hline \multirow{2}{*}{ Items } & \multicolumn{2}{|c|}{ Mild } & \multicolumn{2}{|c|}{ Moderate } & \multicolumn{2}{|c|}{ Severe } \\
\hline & IVM & NIVM & IVM & NIVM & IVM & NIVM \\
\hline \multicolumn{7}{|l|}{ Maternal age } \\
\hline $\begin{array}{l}\text { Mean } \\
\text { (Range) }\end{array}$ & $\begin{array}{c}29.27 \pm 4.84 \\
(21-44)\end{array}$ & $\begin{array}{c}28.35 \pm 4.12 \\
(19-44)\end{array}$ & $\begin{array}{c}28.46 \pm 4.55 \\
(20-39)\end{array}$ & $\begin{array}{c}26.97 \pm 3.96 \\
(20-35)\end{array}$ & $\begin{array}{c}28.83 \pm 5.32 \\
(22-41)\end{array}$ & $\begin{array}{c}27.41 \pm 3.45 \\
(20-35)\end{array}$ \\
\hline \multicolumn{7}{|c|}{ Gestational age at diagnosis } \\
\hline $\begin{array}{l}\text { Mean } \\
\text { (Range) }\end{array}$ & $\begin{array}{c}25.08 \pm 3.88 \\
(17-34)\end{array}$ & $\begin{array}{c}26.93 \pm 4.41 \\
(17-37)\end{array}$ & $\begin{array}{c}29.35 \pm 4.05 \\
(22-37)\end{array}$ & $\begin{array}{c}27.56 \pm 4.52 \\
(19-37)\end{array}$ & $\begin{array}{c}30.74 \pm 5.39 \\
(20-39)\end{array}$ & $\begin{array}{c}29.14 \pm 5.31 \\
(18-39)\end{array}$ \\
\hline \multicolumn{7}{|c|}{ Ventricular Width } \\
\hline $\begin{array}{l}\text { Left, Mean } \\
\text { (Range) }\end{array}$ & $\begin{array}{c}9.85 \pm 1.57 \\
(5.1-11.7)\end{array}$ & $\begin{array}{c}9.88 \pm 1.82 \\
(3.6-11.9)\end{array}$ & $\begin{array}{c}12.27 \pm 1.34 \\
(8.5-14)\end{array}$ & $\begin{array}{c}12.51 \pm 1.65 \\
(8.3-14.9)\end{array}$ & $\begin{array}{c}13.78 \pm 3.24 \\
(8-18)\end{array}$ & $\begin{array}{c}18.08 \pm 6.81 \\
(9.6-42.8)\end{array}$ \\
\hline $\begin{array}{l}\text { Right, Mean } \\
\text { (Range) }\end{array}$ & $\begin{array}{c}8.99 \pm 2.05 \\
(4.1-11.9)\end{array}$ & $\begin{array}{c}9.06 \pm 2.06 \\
(4.5-11.9)\end{array}$ & $\begin{array}{c}10.78 \pm 1.83 \\
(6.5-14.3)\end{array}$ & $\begin{array}{c}11.59 \pm 2.24 \\
(3.4-14.4)\end{array}$ & $\begin{array}{c}17.62 \pm 3.36 \\
(12-22)\end{array}$ & $\begin{array}{c}16.93 \pm 6.35 \\
(7-37.9)\end{array}$ \\
\hline \multicolumn{7}{|c|}{ Ventriculomegaly } \\
\hline Bilateral & 10 & 24 & 11 & 23 & 9 & 21 \\
\hline Unilateral & 34 & 83 & 15 & 7 & 3 & 1 \\
\hline
\end{tabular}

Table 2. The chromosomal anomalies of fetuses and outcomes

\begin{tabular}{|c|c|c|c|c|c|}
\hline \multirow{2}{*}{ Items } & \multicolumn{5}{|c|}{ Cases } \\
\hline & 1 & 2 & 3 & 4 & 5 \\
\hline Ventricular Width $(\mathrm{mm})$ & Mild & Mild & Mild & Moderate & Severe \\
\hline Left & 9.9 & 10.3 & 9.8 & 14 & 16.4 \\
\hline Right & 10.1 & 9.6 & 10.7 & 13.7 & 12.5 \\
\hline $\begin{array}{l}\text { Structural anomalies } \\
\text { through ultrasound }\end{array}$ & $\begin{array}{l}\text { Abnormal ventricular } \\
\text { echo }\end{array}$ & Skeletal dysplasia & Hypoplastic Heart & Skeletal dysplasia & $\begin{array}{c}\text { Multiple } \\
\text { Malformations }\end{array}$ \\
\hline Serum screening & Low Risk & Low Risk & - & - & - \\
\hline Fetal Karyotype & $47, X Y,+\operatorname{mar}[10] / 46$ & $47, X Y,+7$ & $\begin{array}{c}\text { Chromosome } 23 \\
\text { anomaly }\end{array}$ & $\begin{array}{l}\text { 46, XY, der(10)t }(8 ; 10) \\
\quad(q 21.2 ; q 24) \text { pat }\end{array}$ & $46, X Y, \operatorname{add}(1)(p 36)$ \\
\hline Outcome & TOP & TOP & Hydrocephalus & TOP & TOP \\
\hline
\end{tabular}

characteristics of the fetuses with ventriculomegaly are reported in Table 1.

Karyotype examinations were performed in 57 cases (23.7\%), and only 5 showed chromosomal anomalies: three cases in Mild VM, one in Moderate VM and one in the Severe VM. Only 2 of the 5 cases underwent serum screening, and both were deemed low risk. In the majority of these five cases, the parents chose TOP; the exception was the parents of the child with the chromosome 23 anomaly, who had hydrocephalus after birth. No statistical significance was found among the three groups. In the cases that did not have karyotype examination, none of them showed syndromes of chromosomal origin. The chromosomal anomalies of fetuses and outcomes are recorded in Table 2.

Only 29 subjects were screened for TORCH infections. Four cases had abnormal findings: 1 case with Rub-IgM, 2 cases with CMV, and 1 case with Toxo. Three of these cases were in the "Mild Ventriculomegaly" group, and one was in the "Moderate Ventriculomegaly" group. The follow-up investigations found that the infant with Rub infection and one infant with CMV recovered.
The mother of the Toxo-infected fetus chose TOP, and one of the CMV cases experienced a severe ventricular septal defect after birth.

Eighty-two cases were isolated (34.0\%), while associated structural abnormalities were found in 159 cases $(66.0 \%)$. Corpus callosum agenesis was found in most cases; abnormal development of the cardiovascular system and other systems was an important component. Ultrasound scans showed structural abnormalities in 107 fetuses (70.9\%) in the "Mild Ventriculomegaly" group, $30(50.6 \%)$ in the "Moderate Ventriculomegaly" group and $22(64.7 \%)$ in the "Severe Ventriculomegaly" group. The differences among the three groups were not statistically significant. The associated abnormalities of fetuses with ventriculomegaly found on prenatal ultrasound are shown in Table 3.

In the follow-up of 241 cases, 91 cases chose TOP, 4 cases died during the neonatal time. Of the families who did not complete the study, 49 ended their follow-ups when the child was 1 month old, 13 ended at 3 months, 10 ended at 6 months, 6 ended at 1 year, and 4 ended at 3 years. However, 64 cases continued until the end of the 
Table 3. Associated abnormalities found on the prenatal ultrasound

\begin{tabular}{|c|c|}
\hline Items & $n$ \\
\hline \multicolumn{2}{|l|}{ Nervous System } \\
\hline Corpus callosum agenesis & 14 \\
\hline Dilation of the posterior fossa pool & 13 \\
\hline Third ventricle enlargement & 5 \\
\hline Cerebellar vermis agenesis or dilation & 7 \\
\hline Choroid layer cyst & 8 \\
\hline Meningomyelocele & 2 \\
\hline Septum pellucidum agenesis & 5 \\
\hline Abnormal ventricular echo & 10 \\
\hline Enlarged lateral fossa & 1 \\
\hline Isolation of the lateral ventricle posterior horn & 5 \\
\hline Cisterna magna enlargement & 2 \\
\hline \multicolumn{2}{|l|}{ Respiratory System Anomaly } \\
\hline Lung cystic adenomatoid malformation & 1 \\
\hline \multicolumn{2}{|l|}{ Cardiovascular System Anomaly } \\
\hline Cardiac hyperechogenicity & 3 \\
\hline Ventricular septal defects & 3 \\
\hline Hypoplastic heart & 2 \\
\hline Cardiac enlargement & 2 \\
\hline Vascular hypoplasia & 2 \\
\hline \multicolumn{2}{|l|}{ Digestive System Anomaly } \\
\hline Ascites & 4 \\
\hline Intestinal hyperechogenicity & 5 \\
\hline Diaphragmatic hernia & 2 \\
\hline Abdominal lump & 2 \\
\hline Umbilical vein and ductus venosus agenesis & 1 \\
\hline Hepatic hyperechogenicity & 1 \\
\hline Abdominal hyperechogenicity & 1 \\
\hline \multicolumn{2}{|l|}{ Urinary System } \\
\hline Cystic kidney diseases & 1 \\
\hline Renal cysts & 2 \\
\hline Pyelectasis & 12 \\
\hline Hydronephrosis & 1 \\
\hline Renal dysplasia & 1 \\
\hline Pelvic Mass & 1 \\
\hline \multicolumn{2}{|l|}{ Skeletal System } \\
\hline Skeletal dysplasia & 16 \\
\hline Strephenopodia & 1 \\
\hline Spina bifida & 3 \\
\hline Spinal vertebral anomaly & 1 \\
\hline Increased nuchal translucency & 4 \\
\hline Abnormal spine and limbs & 1 \\
\hline \multicolumn{2}{|l|}{ Placental Anomaly } \\
\hline Accessory placenta & 1 \\
\hline Globular placenta & 1 \\
\hline Placental chorioangioma & 1 \\
\hline Polyhydramnios & 5 \\
\hline Multiple Malformations & 6 \\
\hline
\end{tabular}

study period, and the included children were between 16 months and 9 years old. The initial study planned to evaluate the states of surviving children at 1 month, 3 months, 6 months, 1 year, 3 years, 6 years, and 9 years of age to examine both short- and long-term outcomes. However, during the follow-up period, some parents asked to quit the study because their children were in good health and some changed their contact information without notice in advance.

In our study, 91 cases opted for TOP. The abortion rate was $19.5 \%(16 / 82)$ in the IVM group and $47.2 \%$ $(75 / 151)$ in the NIVM group. The difference in the abortion rates between the two groups was significant $(p=0.002)$. In the IVM group, 3 cases in Mild VM, 9 cases in Moderate VM, and 4 cases in Severe VM chose TOP and statistical significance was found in abortion rate among Mild VM, Moderate VM and Severe VM $(p=0.038)$. It was also found that Moderate VM had a higher abortion rate than Mild VM $(p=0.014)$, while no statistical significance was found between Moderate VM and Severe VM. In the NIVM group, 43 cases in Mild VM, 17 cases in Moderate VM, and 15 cases in Severe VM chose TOP and no statistical significance was found among Mild VM, Moderate VM and Severe VM. The data are shown in Table 4.

Of the 150 cases that continued the pregnancy, 20 infants showed significant abnormalities after delivery including four infant deaths during the early neonatal period with NIVM. Of the surviving fetuses, 7 with IVM and 9 with NIVM showed significant abnormalities. Since the outcomes of NIVM mainly depend on the associated malformations, only the outcomes of IVM were used for further data analysis. When IVM were the only findings in the prenatal examinations, 3 cases in the Mild Group, 1 case in the Moderate Group, 3 cases in the Severe Group experienced significant abnormalities after delivery. Statistical significance was found among three groups $(p=0.031)$.The prognoses of Mild Group and Moderate Group had no statistical significance ( $p$ $=1.000$ ), while Moderate Group had more favorable prognoses than Severe Group $(p=0.044)$. These abnormalities are shown in Table 4.

Table 4. The outcomes for the IVM and NIVM groups

\begin{tabular}{|c|c|c|c|c|c|c|}
\hline \multirow{2}{*}{ Items } & \multicolumn{2}{|r|}{ Mild } & \multicolumn{2}{|c|}{ Moderate } & \multicolumn{2}{|c|}{ Severe } \\
\hline & IVM & NIVM & IVM & NIVM & IVM & NIVM \\
\hline Normality & 38 & 55 & 16 & 12 & 5 & 4 \\
\hline Postnatal Death & 0 & 2 & 0 & 0 & 0 & 2 \\
\hline Hydrocephalus & 1 & 2 & 0 & 0 & 1 & 0 \\
\hline Hearing Loss & 1 & 0 & 0 & 0 & 0 & 0 \\
\hline Strabismusand Hearing Loss & 0 & 0 & 0 & 0 & 2 & 0 \\
\hline Hydronephrosis & 0 & 1 & 0 & 0 & 0 & 1 \\
\hline Optic Nerve Abnormalities & 0 & 1 & 0 & 0 & 0 & 0 \\
\hline Other Abnormalities & $\begin{array}{l}1 \text { case-Pachygyria } \\
\text { deformity }\end{array}$ & $\begin{array}{l}1 \text { case-Vascular tumor; } 1 \text { case-PDA, } \\
\text { myopia, amblyopia; } 1 \text { case-VSD }\end{array}$ & $\begin{array}{l}1 \text { case-Mental } \\
\text { retardation }\end{array}$ & $\begin{array}{l}1 \text { case-Pelvic } \\
\text { mass }\end{array}$ & 0 & 0 \\
\hline TOP & 3 & 43 & 9 & 17 & 4 & 15 \\
\hline Total & 44 & 107 & 26 & 30 & 12 & 22 \\
\hline
\end{tabular}


Table 5. Outcome of the fetuses according to the interuterine evaluation of ventricular width

\begin{tabular}{|c|c|c|c|c|}
\hline \multirow{2}{*}{ Evaluation of ventriculomegaly } & \multicolumn{2}{|c|}{ IVM } & \multicolumn{2}{|c|}{ NIVM } \\
\hline & Normalities & Abnormalities & Normalities & Abnormalities \\
\hline \multicolumn{5}{|l|}{ Regeressed in } \\
\hline Mild & 15 & 1 & 15 & 0 \\
\hline Modereate & 6 & 0 & 4 & 0 \\
\hline Severe & 1 & 0 & 0 & 0 \\
\hline \multicolumn{5}{|l|}{ Stable in } \\
\hline Mild & 23 & 2 & 40 & 9 \\
\hline Modereate & 10 & 1 & 8 & 1 \\
\hline Severe & 4 & 3 & 4 & 3 \\
\hline Total & 59 & 7 & 71 & 13 \\
\hline
\end{tabular}

In the ultrasound scan after diagnosis, the width of ventricular dilation regressed in 42 cases and remained the same in 108 cases. Thirty-one cases of "Mild Ventriculomegaly" regressed to the norm during pregnancy; however, 1 infant showed right hearing loss after delivery. In the "Moderate Ventriculomegaly" group, 9 cases regressed to normal, 1 case regressed to "Mild Ventriculomegaly", and none of the babies showed any abnormalities after delivery. In the "Severe Ventriculomegaly" group, the width regressed in 1 case $(16 \mathrm{~mm}, 18 \mathrm{~mm} v s .14 \mathrm{~mm}, 16 \mathrm{~mm})$, with normal a development level after delivery.

Eleven cases (14.9\%) of "Mild VM", 2 cases (10.0\%) of "Moderate VM", and 6 cases (42.9\%) of "Severe VM" showed significant abnormalities after delivery despite a lack of change in their ventriculomegaly during the pregnancy. Better outcomes were found in the regressed group than the unchanged group ( $p=$ 0.026). In the IVM group, no statistical significance were found in outcomes between the regressed group and the unchanged group in fetuses with Mild VM, as did the Moderate VM and Severe VM. In the NIVM group, no statistical significance was found in outcomes between the regressed group and the unchanged group in fetuses with ventriculomegaly of different degrees, respectively. The outcome of fetuses according to the intrauterine evaluation are summarized in Table 5 .

\section{Discussion}

Ventriculomegaly is among the most common cerebral abnormalities found on ultrasound scans during pregnancy. It is defined as lateral ventricles with an atrial diameter of $10 \mathrm{~mm}$ or more. In Cardoza et al.'s study (1), the atrium of the lateral ventricles remained relatively stable between 15 and 40 weeks of gestation $(7.6 \pm 0.6 \mathrm{~mm})$. The criteria for ventriculomegaly first came up in Kramer et al. (2), who recommended $10 \mathrm{~mm}$ as the upper limit of the atrium of the lateral ventricles. Atrial widths greater than $15 \mathrm{~mm}$ were considered "Severe Ventriculomegaly", while atrial widths between $10 \mathrm{~mm}$ and $15 \mathrm{~mm}$ were considered "Mild Ventriculomegaly" with the support a subsequent study (6). However, another study (7) found that the fetal abnormalities surged ( $4 \%$ vs. $14 \%$ ) when the atrial width was consistent with "Mild" (10.0-11.9 mm) or "Moderate Ventriculomegaly" (12.0-14.9 mm); consequently, these studies suggested that ventriculomegaly should be classified as "Mild Ventriculomegaly" (10.0-11.9 mm), "Moderate Ventriculomegaly" (12.0-14.9 mm), and "Severe Ventriculomegaly" ( $\geq 15.0 \mathrm{~mm})$. However, in our study, no statistical significance was found between Mild VM and Moderate VM, thus supporting Krammer et al.'s criteria for ventriculomegaly.

In our study, the mean gestational age at diagnosis was 27 weeks (range: 18-40 weeks). This finding was similar to the findings of Kennelly et al. (8), who reported that the first VM diagnosis occurred at 26.9 weeks gestational age (19-40 weeks); Breeze's study (9) also reported that severe VM was first diagnosed at 28 weeks gestational age (16-36 weeks). However, Baffero et al. (10) reported an earlier gestational age at first diagnosis: 25.1 weeks. The differences might arise from the studies' different compositions; no severe ventriculomegaly cases were included in the Baffero et al. study, and 34 severe VM cases (14.1\%) were included in our study. The mean maternal age in our study was 28 years, which was similar to that of the Gomez et al. study (29 years) (11); however, Kutuk et al. (12) reported a younger age of 26.28 years. Otherwise, all researchers reported the mean finding period was the late period of the second trimester.

The specific etiology of fetal ventriculomegaly is still unclear. It can arise from numerous causes, including brain volume loss, CSF flow obstruction, or CNS malformations, and in some cases, no cause can be determined even after delivery. Several studies have associated fetal ventriculomegaly with chromosomal abnormalities, especially trisomy 21 . The rate of chromosomal abnormalities among cases of fetal ventriculomegaly ranges from 4 to $14 \%(4,13-15)$. In our study, 57 cases underwent karyotype examination, and 5 cases were abnormal $(8.7 \%)$, similar to Chiu et al.'s (16) finding of $9.8 \%$. However, 2 out of the 5 abnormal cases had normal serum screenings for Down syndrome, which suggested the need for a karyotype examination 
using "ventriculomegaly" as an independent factor. Additionally, no statistically significant differences in the rate of chromosomal abnormalities was found among the three groups; this suggests that the degree of ventriculomegaly is not a predictor of aneuploidies, a finding supported by Gaglioti et al.'s study (17). In our study, 16 cases had a history of adverse pregnancy events, including spontaneous abortion and infantile hydrocephalus. Oacute et al. (18) also reported that 32\% of their cases (72/230) had a positive history of adverse pregnancy events, and $2.61 \%(6 / 230)$ had a history of infantile ventriculomegaly, which might indicate that ventriculomegaly is a disease with genetic susceptibility. Therefore, the karyotype examination is of great importance in the counseling process, regardless of the results of the serum screen.

When VM is diagnosed, it is important to consider karyotype abnormalities, intrauterine infections, associated anomalies, the progression of the ventricular width during pregnancy, and the cause of the ventricular dilatation because the prognosis is strongly related to such factors (19).

In utero infections, especially toxoplasmosis and cytomegalovirus, could be a cause of VM. The rate of infection is $10-20 \%$ in cases of severe VM and $0-5 \%$ in cases of mild VM $(5,10,20)$. In our study, 1 subject was positive for Rub, 2 subjects were positive for $\mathrm{CMV}$, and 1 subject was positive for Toxo on the TORCH infections screening. The small number of people who chose to undergo TORCH screening may contribute to the differences, but the outcomes of the infected fetuses were relatively severe compared with those who experienced maternal seroconversion. The infection during pregnancy, particularly TORCH would be related to the enlargement of lateral ventricular width, so the screening for TORCH should also be recommended in the process of consultation with parents.

The overall rate of associated structural malformations was $62.7 \%$ in our study; however, in previous studies, the rates range widely, from $10 \%$ to $77.4 \%$. Corpus callosum anomalies were the most frequently found malformations in our study, as in the previous studies $(9,17,21-24)$. In our study, 107 fetuses (70.9\%) with "Mild Ventriculomegaly" and 30 (50.6\%) with "Moderate Ventriculomegaly" had associated abnormalities. Twenty-two fetuses (64.7\%) with "Severe Ventriculomegaly" had associated anomalies; this rate is similar to the $65 \%$ reported by Breeze et al.'s (9), but higher than the $58 \%$ reported by Gaglioti et al. (17). Previous study has also suggested that the rate of associated anomalies increased from $41 \%$ to $76 \%$ when the atrial width increased from $10-12.0 \mathrm{~mm}$ to $12-14.9$ $\mathrm{mm}$ (17); however, we found no differences among the three groups in our study, and a recent study supports our findings (10). The different general populations of the studies might explain most of the differences, and improvements in prenatal imaging technology could increase the rates of associated abnormalities.

Our study suggested that the parents in the NIVM group chose TOP more often than those in the IVM group and the associated structural malformations might be the cause. In the IVM group, when ventricular width is above $12 \mathrm{~mm}$, the abortion ratio rose and suggested that the degree of ventriculomegaly might be the main cause for parents to choose TOP when the ventriculomegaly is isolated. In the NIVM group, no significance was found in different degrees, which suggested that the structural malformations rather than the ventriculomegaly itself affected the parents to choose TOP.

The outcomes of NIVM were mainly determined by associated structures of fetuses, and the outcomes of IVM were used for further data analysis. In our study, the outcomes for the "Mild VM" and "Moderate VM" groups were significantly better than those of the "Severe VM" group. Our study suggests that the prognoses of fetuses with VM were strongly determined by the extent of ventricular dilation, a finding that is supported by previous studies.

Signorelli et al. (3) reported 60 cases of mild ventriculomegaly, and none had poor prognoses; thus, the researchers concluded that mild ventriculomegaly was a variation, and subsequent studies have supported this conclusion $(25,26)$. However, Kutuk et al. (12) reported that 9 out of 25 cases with mild VM showed mental or locomotor activity retardation, and some studies have supported these findings (11,27-29). In our study, 3 cases in the "Mild VM" group and 1 case in the "Moderate VM" group had poor neurodevelopmental prognoses, which suggests that although fetuses with "Mild VM" may have more favorable outcomes than fetuses with severe ventriculomegaly, they still faced the possibility of developing an abnormality after delivery; thus, ultrasound scans and other examinations are still essential during pregnancy and after birth.

A ventricular atrial width greater than $15 \mathrm{~mm}$ is an indicator of poor prognosis associated with abnormalities and high mortality rates $(9,17,26,30)$. Gaglioti et al. (17) reported poor prognoses in $27.5 \%$ of the "Severe ventriculomegaly" group, while Weichert et al. (5) reported poor prognoses in $40.6 \%$. In our study, the incidence of abnormalities was $40.0 \%$ in the "severe ventriculomegaly" group, and $13.3 \%$ of the pregnancies in that group ended in neonatal death. Different followup criteria and high rates of TOP in our study might explain the differences between our results and those of other studies. However, both our study and others indicated that severe ventriculomegaly may have a poorer prognosis and should receive more attention in clinical practice.

The progression of ventricular width was an indicator of a possible unfavorable prognosis. In previous studies, most of the fetuses had a more favorable outcome when the ventriculomegaly regressed during pregnancy; however, Hannon et al. 
(30) claimed that atrial measurements did not predict neonatal death, and Falip et al. (6) observed poor neurological outcomes in infants with all types of ventriculomegaly: stable, regressive and resolved. In our study, 42 cases showed a regression of ventricular width, and only one of these cases had an abnormality after delivery, which was similar to the findings of a previous study (31). No progression of ventricular width was observed, perhaps because of the large proportion of mothers who chose TOP. Of the 108 cases whose ventricular width remained the same, 19 developed associated abnormalities. The differences in abnormalities between the groups with stable and regressed ventricular with was statistically significant, suggesting that the outcomes of fetuses with VM were related to the evolution of ventriculomegaly in utero, and the prognoses were better when ventriculomegaly regressed or even disappeared. However, in the IVM group, no statistical significance was found in outcomes between the regressed group and the unchanged group in fetuses with Mild VM, which might suggest that the evolution of ventricular width was not an important factor that was related to the prognoses of fetuses with mild isolated ventriculomegaly. In the IVM group, we also failed to find statistical significance in outcomes between the regressed group and the unchanged group in Moderate VM and Severe VM probably due to the small number in each group. Otherwise, our study suggested that changes in ventricular width of the fetus are essential, and additional ultrasound scans during pregnancy are of great importance.

There were limitations in our study. The main limitation is the limited examination of TORCH and karyotype examinations might result in a bias for the categories of IVM and NIVM. TORCH was usually recommended for our patients but only after their approval could it be done and all the patients that had a history of pet contact had done the tests. Karyotype examinations were only performed in little parts of patients but none of the newborns showed signs of chromosomal origins. Second, MRIs were only performed in a few cases for technical restrictions. MRI is of good value in the diagnosis of ventriculomegaly and other intracranial structural abnormalities, but it was not until recent years that MRI was introduced into our hospital and the data was not sufficient. However, MRI data are expected to be collected and included in our further studies as evaluation indicators of children's statuses.

In conclusion, the degree and progression of ventricular dilatation are mean factors that affect the pregnancy outcomes and prognoses of fetal ventriculomegaly. When VM is diagnosed in utero, accurate examinations to exclude associated anomalies should be performed. Prenatal diagnosis is necessary even when serum screening results are negative. TORCH screening should also be suggested, and follow-up is of great importance. Because the ideal follow up work was too hard to do, there were many limitations and areas falling short in our work. Multicenter studies and critical follow-up should be planned to continue to do this meaningful work.

\section{Acknowledgements}

This work was supported by the National Natural Science Foundation of China No. 31571196 (to Ling Wang), the Science and Technology Commission of Shanghai Municipality 2015 YIXUEYINGDAO project No. 15401932200 (to Ling Wang), the FY2008 JSPS Postdoctoral Fellowship for Foreign Researchers P08471 (to Ling Wang), the National Natural Science Foundation of China No. 30801502 (to Ling Wang), the Shanghai Pujiang Program No. 11PJ1401900 (to Ling Wang), and Development Project of Shanghai Peak DisciplinesIntegrated Chinese and Western Medicine No.20150407.

\section{References}

1. Cardoza JD, Goldstein RB, Filly RA. Exclusion of fetal ventriculomegaly with a single measurement: The width of the lateral ventricular atrium. Radiology. 1988; 169:711-714.

2. Kramer RL, Yaron Y, Johnson MP, Evans MI, Treadwell MC, Wolfe HM. Differences in measurements of the atria of the lateral ventricle: Does gender matter? Fetal Diagn Ther. 1997; 12:304-305.

3. Signorelli M, Tiberti A, Valseriati D, Molin E, Cerri V, Groli C, Bianchi UA. Width of the fetal lateral ventricular atrium between 10 and $12 \mathrm{~mm}$ : A simple variation of the norm? Ultrasound Obstet Gynecol. 2004; 23:14-18.

4. Sethna F, Tennant PW, Rankin J, S CR. Prevalence, natural history, and clinical outcome of mild to moderate ventriculomegaly. Obstet Gynecol. 2011; 117:867-876.

5. Weichert J, Hartge D, Krapp M, Germer U, Gembruch U, Axt-Fliedner R. Prevalence, characteristics and perinatal outcome of fetal ventriculomegaly in 29,000 pregnancies followed at a single institution. Fetal Diagn Ther. 2010; 27:142-148,

6. Falip C, Blanc N, Maes E, Zaccaria I, Oury JF, Sebag G, Garel C. Postnatal clinical and imaging follow-up of infants with prenatal isolated mild ventriculomegaly: A series of 101 cases. Pediatr Radiol. 2007; 37:981-989.

7. Pilu G, Falco P, Gabrielli S, Perolo A, Sandri F, Bovicelli L. The clinical significance of fetal isolated cerebral borderline ventriculomegaly: Report of 31 cases and review of the literature. Ultrasound Obstet Gynecol. 1999; 14:320-326.

8. Kennelly MM, Cooley SM, McParland PJ. Natural history of apparently isolated severe fetal ventriculomegaly: Perinatal survival and neurodevelopmental outcome. Prenat Diagn. 2009; 29:1135-1140.

9. Breeze AC, Alexander PM, Murdoch EM, MissfelderLobos HH, Hackett GA, Lees CC. Obstetric and neonatal outcomes in severe fetal ventriculomegaly. Prenat Diagn. 2007; 27:124-129.

10. Baffero GM, Crovetto F, Fabietti I, Boito S, Fogliani R, Fumagalli M, Triulzi F, Mosca F, Fedele L, Persico N. 
Prenatal ultrasound predictors of postnatal major cerebral abnormalities in fetuses with apparently isolated mild ventriculomegaly. Prenat Diagn. 2015; 35:783-788.

11. Gomez-Arriaga P, Herraiz I, Puente JM, ZamoraCrespo B, Nunez-Enamorado N, Galindo A. Midterm neurodevelopmental outcome in isolated mild ventriculomegaly diagnosed in fetal life. Fetal Diagn Ther. 2012; 31:12-18.

12. Kutuk MS, Ozgun MT, Uludag S, Dolanbay M, Poyrazoglu HG, Tas M. Postnatal outcome of isolated, nonprogressive, mild borderline fetal ventriculomegaly. Childs Nerv Syst. 2013; 29:803-808.

13. Ouahba J, Luton D, Vuillard E, Garel C, Gressens P, Blanc N, Elmaleh M, Evrard P, Oury JF. Prenatal isolated mild ventriculomegaly: Outcome in 167 cases. BIOG. 2006; 113:1072-1079.

14. Tatli B, Ozer I, Ekici B, Kalelioglu I, Has R, Eraslan E, Yuksel A. Neurodevelopmental outcome of 31 patients with borderline fetal ventriculomegaly. Clin Neurol Neurosurg. 2012; 114:969-971.

15. Lam SJ, Kumar S. Evolution of fetal ventricular dilatation in relation to severity at first presentation. J Clin Ultrasound. 2014; 42:193-198.

16. Chiu TH, Haliza G, Lin YH, Hung TH, Hsu JJ, Hsieh TT, Lo LM. A retrospective study on the course and outcome of fetal ventriculomegaly. Taiwan J Obstet Gynecol. 2014; 53:170-177.

17. Gaglioti P, Danelon D, Bontempo S, Mombro M, Cardaropoli S, Todros T. Fetal cerebral ventriculomegaly: Outcome in 176 cases. Ultrasound Obstet Gynecol. 2005; 25:372-377.

18. Joo JG, Toth Z, Beke A, Papp C, Toth-Pal E, Csaba A, Szigeti Z, Rab A, Papp Z. Etiology, prenatal diagnostics and outcome of ventriculomegaly in 230 cases. Fetal Diagn Ther. 2008; 24:254-263.

19. D'Addario V, Pinto V, Di Cagno L, Pintucci A. Sonographic diagnosis of fetal cerebral ventriculomegaly: An update. J Matern Fetal Neonatal Med. 2007; 20:7-14.

20. D'Addario V, Rossi AC. Neuroimaging of ventriculomegaly in the fetal period. Semin Fetal Neonatal Med. 2012; 17:310-318

21. Madazli R, Sal V, Erenel H, Gezer A, Ocak V. Characteristics and outcome of 102 fetuses with fetal cerebral ventriculomegaly: Experience of a university hospital in Turkey. J Obstet Gynaecol. 2011; 31:142-145.
22. Melchiorre K, Bhide A, Gika AD, Pilu G, Papageorghiou AT. Counseling in isolated mild fetal ventriculomegaly. Ultrasound Obstet Gynecol. 2009; 34:212-224.

23. Gaglioti P, Oberto M, Todros T. The significance of fetal ventriculomegaly: Etiology, short- and long-term outcomes. Prenat Diagn. 2009; 29:381-388.

24. Parazzini C, Righini A, Doneda C, Arrigoni F, Rustico M, Lanna M, Triulzi F. Is fetal magnetic resonance imaging indicated when ultrasound isolated mild ventriculomegaly is present in pregnancies with no risk factors? Prenat Diagn. 2012; 32:752-757.

25. Tugcu AU, Gulumser C, Ecevit A, Abbasoglu A, Uysal NS, Kupana ES, Yanik FF, Tarcan A. Prenatal evaluation and postnatal early outcomes of fetal ventriculomegaly. Eur J Paediatr Neurol. 2014; 18:736-740.

26. Atad-Rapoport M, Schweiger A, Lev D, Sadan-Strul S, Malinger G, Lerman-Sagie T. Neuropsychological followup at school age of children with asymmetric ventricles or unilateral ventriculomegaly identified in utero. Bjog. 2015; 122:932-938

27. Lyall AE, Woolson S, Wolfe HM, Goldman BD, Reznick JS, Hamer RM, Lin W, Styner M, Gerig G, Gilmore JH. Prenatal isolated mild ventriculomegaly is associated with persistent ventricle enlargement at ages 1 and 2. Early Hum Dev. 2012; 88:691-698.

28. Ball JD, Abuhamad AZ, Mason JL, Burket J, Katz E, Deutsch SI. Clinical outcomes of mild isolated cerebral ventriculomegaly in the presence of other neurodevelopmental risk factors. J Ultrasound Med. 2013; 32:1933-1938

29. Gilmore JH, Smith LC, Wolfe HM, Hertzberg BS, Smith JK, Chescheir NC, Evans DD, Kang C, Hamer RM, Lin W, Gerig G. Prenatal mild ventriculomegaly predicts abnormal development of the neonatal brain. Biol Psychiatry. 2008; 64:1069-1076.

30. Hannon T, Tennant PW, Rankin J, Robson SC. Epidemiology, natural history, progression, and postnatal outcome of severe fetal ventriculomegaly. Obstet Gynecol. 2012; 120:1345-1353.

31. Parilla BV, Endres LK, Dinsmoor MJ, Curran L. In utero progression of mild fetal ventriculomegaly. Int J Gynaecol Obstet. 2006; 93:106-109.

(Received March 24, 2016; Revised April 7, 2016; Accepted April 8, 2016) 